\title{
Solar model: Element diffusion or metal-enhanced envelope?
}

\author{
J. Y. Yang, Y. Li, and H. Y. Xu \\ Yunnan Observatory, NAOCAS, PO Box 110, Kunming 650011, PR China \\ e-mail: gtsa@public.km.yn.cn \\ Received 16 December 1998 / Accepted 4 October 2000

\begin{abstract}
Solar models with moderate enrichment of heavy elements in the convective envelope (CSM) and with element diffusion (DSM) are investigated using updated input physics. It is found that both CSM and DSM can result in adequate depth of the convection zone and appropriate surface helium abundance, and the agreement between the calculated and observed p-mode frequencies are also improved. The sound speed of DSM is better than CSM and the standard solar model (SSM) in the deep interior, which means that element diffusion helps improve the sound speed profile in the solar interior. In the area just below the base of the convection zone, CSM is the best of the three models.
\end{abstract}

Key words. solar structure - solar evolution - solar oscillations

\section{Introduction}

Solar models are crucial to verify the hypotheses used in modeling of stellar evolution. Precise solar models have been constructed over the past three decades. Today we have two methods to probe the structure of solar interior: solar neutrino experiments and helioseismological analysis. The results of these investigations impose stringent restrictions on the solar modeling, and allow definitive tests and refinements of the input physics.

An important assumption involved in the so-called standard solar models (SSM) is that, except for the central nuclear transmutation, the chemical composition is homogeneous in the solar interior and remains constant throughout the solar evolution. A discrepancy has existed between SSM predictions of neutrino fluxes and the rates observed in terrestrial experiments for more than three decades (Bahcall 1989). In recent years, the combined results from four solar neutrino experiments have sharpened the discrepancy. Low $Z$ models were proposed at the beginning to reduce the predicted solar neutrino flux (Bahcall \& Ulrich 1971). Contrary to the standard models, low $Z$ models take into account the possibility of chemical stratification of the Sun. A pre-main sequence star is chemically homogeneous, since it experiences fully convective mixing during the Hayashi phase of evolution. However, the star may capture afterwards some interplanetary matter. Joss (1974) suggested that stellar surfaces may be continuously enriched with heavy elements by infalling comets. Levy \& Ruzmaikina (1994) investigated the effects of dust-gas separation during the star formation

Send offprint requests to: J. Y. Yang process. As they pointed out, dust grains that aggregate by turbulence to large sizes will settle from the upper layers toward the center of the molecular cloud and merge into the proto-planetary nebula disk, and finally fall onto the star by means of disk accretion. For the case of the Sun that has a convection zone just below its photosphere, the falling matter will be mixed into the whole convection zone. As the metal abundance of the interplanetary dust is much higher than that of the solar material, the solar convection zone will be enhanced with heavy elements.

Low $Z$ models can give rather low neutrino fluxes, but can not completely solve the solar neutrino problems. Moreover, they usually result in shallow convection zones and very low initial helium abundance, and their calculated p-mode oscillation frequencies and sound speed in the solar interior are not in agreement with observations (Christensen-Dalsgaard et al. 1979; Christensen-Dalsgaard \& Gough 1980; Bahcall \& Ulrich 1988). Therefore, in recent years low $Z$ models have been thought to be unrealistic. At the same time, more and more authors prefer the standard solar models with helium and heavy element diffusion (Bahcall \& Pinsonneault 1992; Bahcall et al. 1995; Bahcall et al. 1997; Bahcall et al. 1998). The computed depth of the convective zone, the present-day surface helium abundance and the sound speeds of these models are in good agreement with helioseismological measurements, but the calculated neutrino event rates are even higher than that of the standard solar models.

However, much evidence indicates that the solar envelope has inevitably been contaminated by interplanetary material, even if not as much as demanded by previous 
low $Z$ models. Henney \& Ulrich (1998) investigated the effects of cometary mass accretion with heavy-element diffusion on solar oscillation frequencies. They found that for solar models with element diffusion, the addition of mass accretion shifts the predicted p-mode frequencies to slightly lower values, and the predicted g-mode frequencies exhibit a greater shift of approximately $1 \mu \mathrm{Hz}$. The net accreted masses they chose are 1.7, 8.3 and 166 earth masses, but we think these values are too low or too high. In this paper, we investigate the moderate enhancement of the envelope metallicity, using updated input physics, and focus our attention on the influences of the structure and p-mode oscillations of the Sun. Our input physics and model computation method are described in Sect. 2. We discuss and compare the properties of the metal-enriched model with that of the standard and diffusion models in Sect. 3 and their seismic properties in Sect. 4. Our main conclusions are summarized in Sect. 5 .

\section{Physics assumptions and computational methods}

Solar evolution models are calculated using an evolution code originally written by Bohdan Paczynski and Maciek Kozlowski, and updated by Ryszard Sienliewicz in 1995. We made some modifications to incorporate metal-enhancement of the solar envelope. Energy transfer by convection is treated according to the standard mixinglength theory, and the boundaries of the convection zones are determined by the Schwarzschild criterion (see Cox \& Giuli 1968). Nuclear reaction rates are those used in BP95 (Bahcall et al. 1995). The equation of state we used here is the OPAL EOS from Lawrence Livermore National Laboratory (Rogers 1994; Rogers et al. 1996). OPAL opacities GN93hz series (Rogers \& Iglesias 1995; Iglesias \& Rogers 1996) are used in the high temperature region. In the outer envelope of the Sun, low-temperature opacities from Alexander \& Ferguson (1994) are used to include the contributions from molecules and grains. These two sets of opacity data are connected around temperatures of $8000 \mathrm{~K}$, at that fitting point they coincide closely. For the model that considered helium and heavy element diffusion, we used the exportable diffusion subroutine of Thoule et al. (Thoule et al. 1994). Our solar models consist of more than 10000 mass zones and are evolved from the zero-age main sequence for 210 time steps to reach the present solar age.

Previous low $Z$ models usually adopted very low central metal abundance, so as to reduce the calculated neutrino emission fluxes. Observations show, however, that the Sun has the normal Population I abundance (Anders \& Grevesse 1989; Grevesse \& Noels 1993). Solar neutrino experiments also put strong restrictions on the metal abundance distribution, i.e., the metal abundances in the solar interior do not deviate too much from its surface observation value (Fukugita \& Hata 1998). Correspondingly, we chose the initial metallicity to be $Z_{0}=0.015$, which is much higher than the choices of other investigations (see Bahcall \& Ulrich 1971; Christensen-Dalsgaard et al. 1979). Interplanetary mass accretion can be very complex. According to the present observation, the processes of mass accretion and mass loss may be co-exist at the same time. If considerable interplanetary matter falls onto the Sun, we prefer to believe that this occurs during the early phase of the solar evolution, when the Sun is still surrounded by an opaque disk. We assume, for simplicity, that the Sun acquires an amount of matter made up of purely heavy elements at a certain moment in its early evolution, resulting in the enhancement of its envelope metallicity to the present observed value of $Z=0.02$. This is also distinct from the previous approaches of gradual enrichment of the envelope metallicity. As we can see below, the mass falling onto the Sun corresponding to this enhancement of metal abundance is about 40 earth masses, which is just in the gap of Henney and Ulrich's investigation. The fallen interplanetary matter immediately spreads out by convective motion to the whole convection zone. The Sun then consists of two parts, the envelope enhanced with heavy elements and the core, being of the original metal abundance, with the bottom of the convection zone as the boundary.

Suppose $f \times M_{\odot}$ to be the initial mass of the model and $k \times f \times M_{\odot}$ the mass of the convection zone just before the metal contamination. After the acquirement of the interplanetary matter, which is assumed to be composed of purely heavy elements, the mass of the model increases to $1 M_{\odot}=1.989110^{33} \mathrm{~g}$, while the metal abundance in the convection zone increases from $Z_{0}$ to $Z$. After a few steps of simple manipulations, $f$ is found to be:

$$
f=\frac{1-Z}{1-Z+k\left(Z-Z_{0}\right)}
$$

\section{Properties of evolution models}

We have calculated three different series of evolution models. SSM and DSM are standard models, without and with helium and heavy element diffusion, respectively. Neither model considers the effects of metal enrichment. CSM is a metal enriched model without element diffusion, in order to distinguish the effects of the envelope metal enrichment and element diffusion. We assume that the metal enrichment takes place at the zero-age main sequence. The initial helium abundance, $Y_{0}$, and the mixing-length to pressure scale height ratio, $\alpha$, are iteratively adjusted to give the solar luminosity $\left(3.84410^{33} \mathrm{ergs} \mathrm{s}^{-1}\right)$ and solar radius $\left(6.9610^{10} \mathrm{~cm}\right)$ at the present solar age $(4.566 \mathrm{Gyrs})$ (see Dar \& Shaviv 1996). In the meantime, the mass fraction of the convection zone just before the interplanetary contamination, $k$, is also found.

Table 1 gives the basic parameters of the three models, where $Y_{0}$ and $Z_{0}$ are the initial helium and metal abundance. For solar age models, $Y_{\mathrm{s}}$ and $Z_{\mathrm{s}}$ are the surface helium and metal abundance, $Y_{\mathrm{c}}$ and $Z_{\mathrm{c}}$ are the central helium and metal abundance, $T_{\mathrm{c}}$ and $\rho_{\mathrm{c}}$ are the central temperature and density, $M_{\mathrm{bc}}$ is the mass interior to the 
Table 1. Properties of evolution models

\begin{tabular}{lllll}
\hline Parameters & SSM & DSM & CSM & Observation \\
\hline$Y_{0}$ & 0.275 & 0.272 & 0.250 & \\
$Z_{0}$ & 0.02 & 0.02 & 0.015 & \\
$\alpha$ & 1.638 & 1.726 & 1.697 & \\
$1-f\left(M_{\odot}\right)$ & & & 0.000129 & \\
$Y_{\text {surf }}$ & 0.275 & 0.242 & 0.248 & $0.249 \pm 0.003$ \\
$Z_{\text {surf }}$ & 0.02 & 0.018 & 0.02 & \\
$Y_{\mathrm{c}}$ & 0.632 & 0.649 & 0.605 & \\
$Z_{\mathrm{c}}$ & 0.02 & 0.021 & 0.015 & \\
$T_{\mathrm{c}}\left(10^{6} \mathrm{~K}\right)$ & 15.69 & 15.79 & 15.35 & \\
$\rho_{\mathrm{c}}\left(\mathrm{g} / \mathrm{cm}^{3}\right)$ & 153.2 & 157.7 & 150.7 & \\
$M_{\mathrm{bc}}\left(M_{\odot}\right)$ & 0.979 & 0.977 & 0.979 & \\
$R_{\mathrm{bc}}\left(R_{\odot}\right)$ & 0.724 & 0.716 & 0.719 & $0.713 \pm 0.001$ \\
$T_{\mathrm{bc}}\left(10^{6} \mathrm{~K}\right)$ & 2.122 & 2.155 & 2.139 & \\
$\Phi_{\mathrm{Cl}}(\mathrm{SNUs})$ & 7.70 & 8.52 & 5.32 & $2.55 \pm 0.25$ \\
$\Phi_{\mathrm{Ga}}(\mathrm{SNUs})$ & 127.50 & 131.52 & 115.88 & $73.4 \pm 5.7$ \\
\hline
\end{tabular}

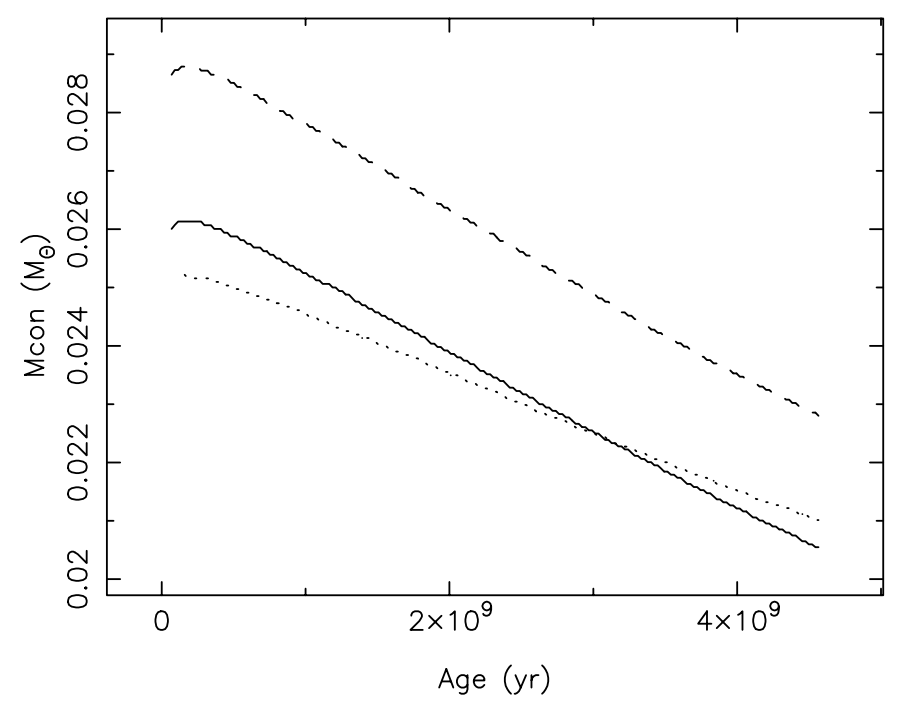

Fig. 1. Masses included beyond the bases of the convection zones of SSM, DSM and CSM during the evolution. Solid line represent SSM, dashed line is for DSM, and dotted line is for CSM

base of the convection zone, $R_{\mathrm{bc}}$ and $T_{\mathrm{bc}}$ are the radius and temperature at the base of the convection zone, $\Phi_{\mathrm{Cl}}$ and $\Phi_{\mathrm{Ga}}$ are neutrino fluxes for $\mathrm{Cl}$ and $\mathrm{Ga}$ detectors.

It can be found from Table 1 that $1-f$, which is the mass in units of $M_{\odot}$ falling onto the Sun, is about $0.00013 M_{\odot}$. This value $\left(10^{29} \mathrm{~g}\right)$ coincides with those of some other authors. For instance, Jeffery et al. (Jeffery et al. 1997) considered six main sources of infalling material to estimate the mass accreted onto the solar surface during its main-sequence lifetime to be about $610^{29} \mathrm{~g}$.

If we take the metal abundance of the interplanetary dust to be the solar value of 0.02 as a minimum limit, the mass required to provide the captured heavy elements is $0.0065 M_{\odot}$, which is about 30 percent of the mass of the solar proto-planetary nebula of $0.02 M_{\odot}$ (Safronov \& Ruzmaikina 1985). Therefore, the accreted mass in our model is acceptable.
It is very interesting to notice that the surface helium abundance and the bases of the convection zone for the metal enriched model CSM are 0.248 and $0.719 R_{\odot}$; both are in good agreement with recent helioseismic determinations $Y_{\mathrm{S}}=0.249 \pm 0.003$ (Basu \& Antia 1997) and $R_{\mathrm{cz}}=(0.713 \pm 0.001) R_{\odot}($ Basu \& Antia 1995). This is contrary to the results of previous investigations, in which metal enriched models result in shallow convection zones and very low surface helium abundances (see ChristensenDalsgaard et al. 1979; Christensen-Dalsgaard \& Gough 1980; Bahcall \& Ulrich 1988). For the standard model with element diffusion, DSM, these two values are 0.242 and $0.716 R_{\odot}$, also in good agreement with the measured values. This is similar to many other authors' results (see Bahcall et al. 1995; Bahcall \& Pinsonneault 1998). We can conclude from Table 1 that SSM is in poor agreement with helioseismological analysis. Element diffusion and settling are initially introduced to reduce the envelope helium abundance during the evolution. Now we have another approach to remedy the discrepancy between the calculated and observed solar envelope helium abundance. It should be noticed that, unlike the helium diffusion that brings the helium originally in the solar envelope down to the solar core and leaves a helium abundance profile in the present solar interior, our approach will reduce the helium abundance over the whole solar interior from the beginning of the solar evolution.

It can be seen from Table 1 that CSM gives lower neutrino fluxes for either $\mathrm{Cl}$ or Ga detectors while DSM gives higher fluxes than SSM. The reason is mainly due to the fact that the central helium abundance of CSM, which is of strong influence on the flux of high energy neutrinos, is considerably lower than that of SSM, while $Y_{\mathrm{c}}$ of DSM is higher than that of SSM. Further decrease of the central heavy element abundance can still reduce the neutrino fluxes, along with smaller values of the initial helium abundance, and therefore, is not a reasonable measure to solve the solar neutrino anomaly.

Evolution for the convection zones of three models are shown in Figs. 1 and 2. The solid line is for SSM, dashed line is for DSM and dotted line is for CSM. As shown in Fig. 1, the masses exterior to the bases of the convection zones for all three models always decrease during their evolution, and DSM has more mass in its convection envelope than CSM and SSM. The temperature at the base of the convection zone for the three models show similar behavior: they all decrease as the age increases, and DSM has a higher temperature than SSM and CSM, as seen in Fig. 2.

Figures 3 and 4 show for the solar age models the differences of the temperature and pressure profiles of DSM and CSM with respect to that of SSM. It can be seen that the temperatures over the whole interior of CSM are 1-3\% cooler than SSM, while DSM has almost the same temperature as SSM in the core and is approximately $2.5 \%$ cooler than SSM in the convective envelope. The pressure of CSM is higher (about $0-1 \%$ ) in the nuclear burning core and lower (about $0-4 \%$ ) in the middle radiative and 


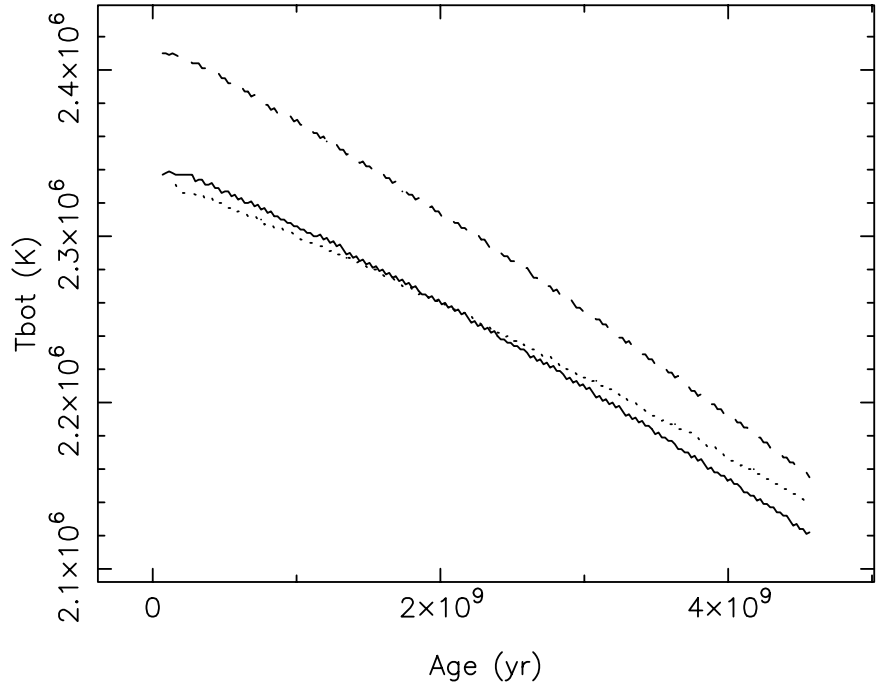

Fig. 2. Temperatures at the bases of the convection zones for SSM, DSM and CSM. Solid line is for SSM, dashed line is for DSM, and dotted line is for CSM

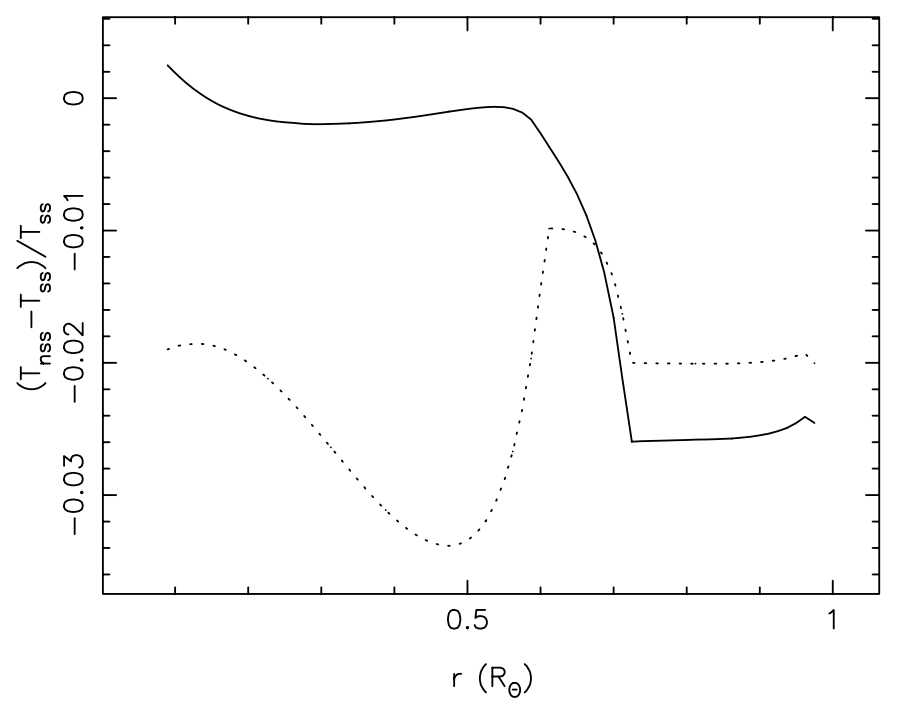

Fig. 3. Temperature differences of DSM and CSM with respect to SSM. Solid line is for DSM to SSM, and dotted line for CSM to SSM

outermost convective zones than that of SSM, while the behave of DSM is opposite, i.e. lower in the core and higher in the envelope.

\section{Frequencies of the p-mode oscillations}

The three solar age models are input into an updated linear adiabatic pulsation code of $\mathrm{Li}$ (1992) to calculate the p-mode oscillation frequencies. We calculated the frequencies for the spherical harmonic degree $l=0,1,2,3$. Frequencies of modes with high values of $l$ are seriously influenced by the non-adiabatic effects and are not considered in this paper. We have used the gray atmosphere in our p-mode oscillation calculations.

In Fig. 5 we compare the calculated frequencies of $l=0,1,2,3$ with the observed values obtained by

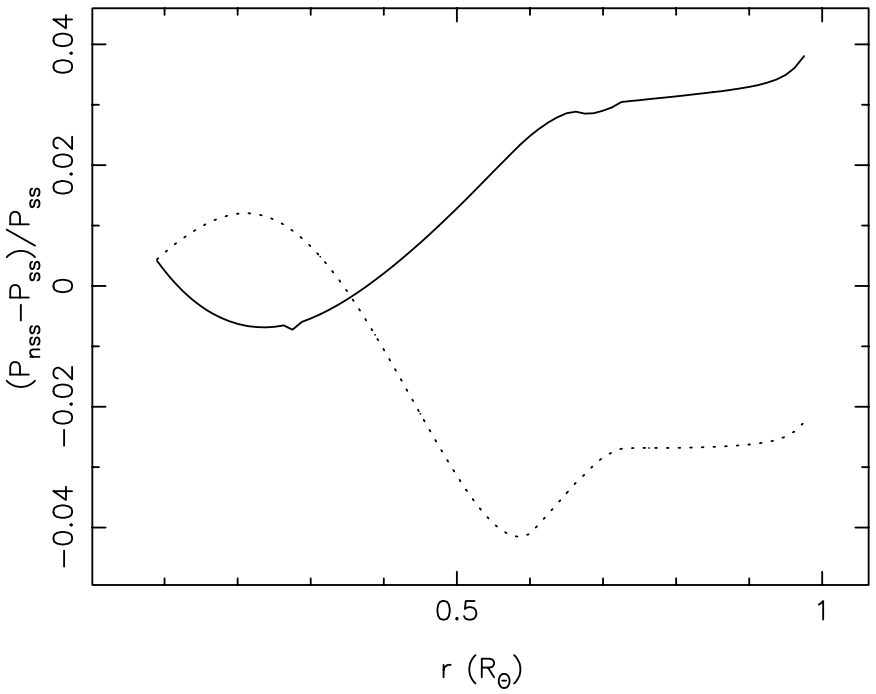

Fig. 4. Pressure differences of DSM and CSM with respect to SSM. Solid line is for DSM to SSM, and dotted line for CSM to SSM

Libbrecht et al. (1990). It is found that the p-mode frequencies of DSM and CSM are in better agreement with the observations than those of SSM. This is an important result, and completely contradicts the conclusions of previous low $Z$ models. As our metal enriched models should not necessarily result in worse p-mode frequencies, a major argument against the possibility of non-homogeneous solar models may no longer be valid. We notice that the tendency of the frequency differences between the calculation and observation is similar for all three models. For the cases of $l=0,1$, the p-mode frequencies of DSM and CSM are in good agreement with each other, while those of SSM are slightly higher than those of DSM and CSM. When $l=2,3$, the p-mode frequencies of CSM are close to those of SSM.

Figure 6 shows the profiles of sound speed differences of the three models with respect to the observed values (Basu et al. 2000). It can be seen from Fig. 6 that the calculated sound speed is higher in the nuclear fusion cores and lower in the outer adjacent radiative zones than the observed sound speed, and is almost the same as the observation in the convection zones for all three models. For the solar interior where $R<0.6 R_{\odot}, \mathrm{DSM}$ is in better agreement with observation than SSM, while CSM is worse than $\mathrm{SSM}$, but the maximum difference is less than $1 \%$. It seems that all three models are acceptable, and element diffusion could improve the sound speed profile in the deep interior of the Sun. In the area just below the base of the convection zone, the calculated sound speed from CSM is almost the same as the observed value, while DSM and SSM give their maximum difference, which is about $0.6 \%$.

\section{Discussions and conclusions}

Low $Z$ models have been rejected so far due to three major disagreements with observations: the depths of their convection zones are shallower than the seismic determined 

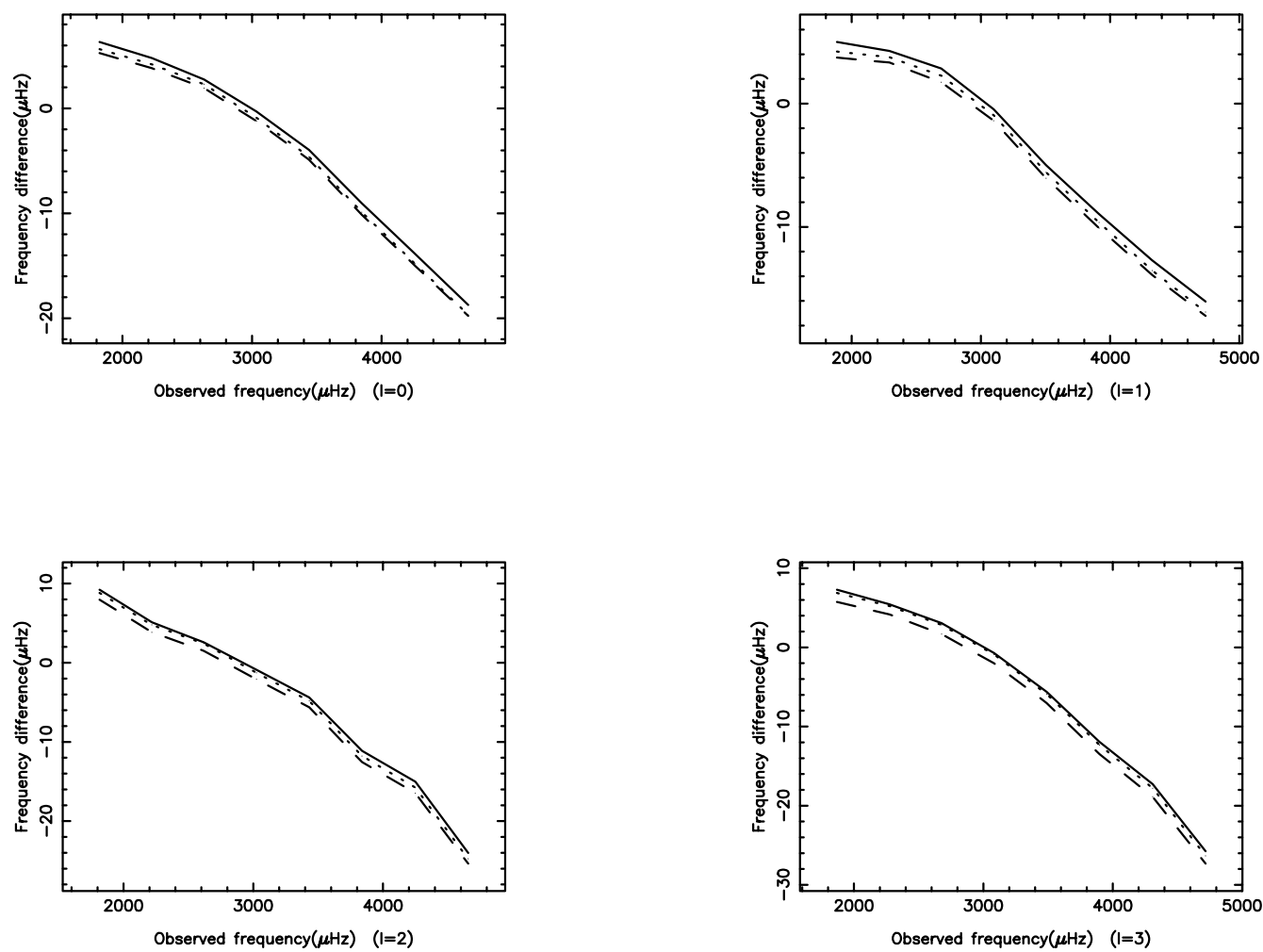

Fig. 5. Comparisons of calculated solar p-mode frequencies of $l=0,1,2,3$ with respect to the observations. Solid line is for SSM, dashed line is for DSM and dotted line is for CSM

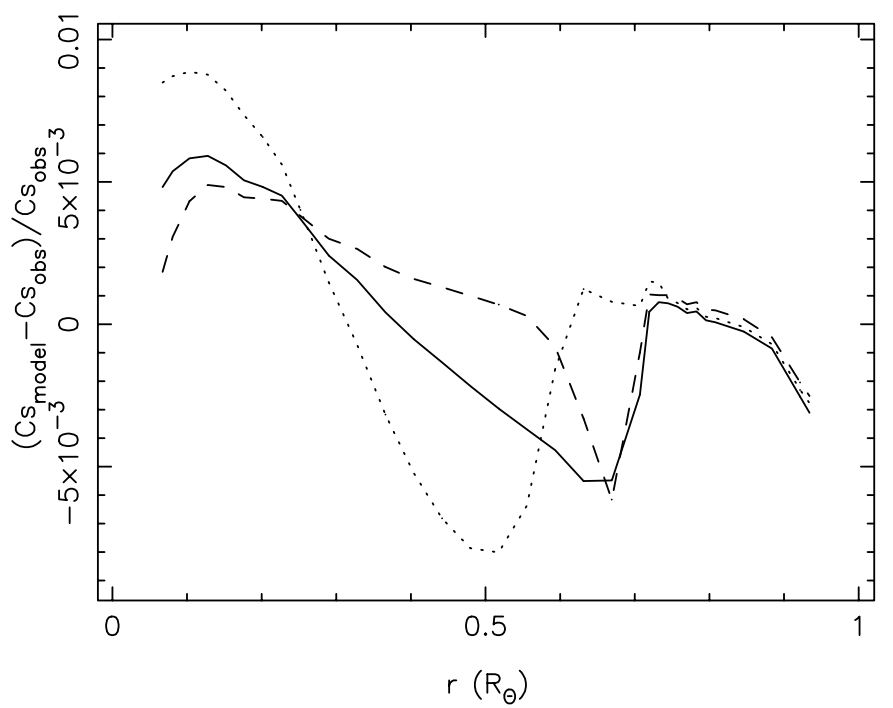

Fig. 6. The sound speed differences of three models with respect to observation values. Solid line is for SSM, dashed line is for DSM and dotted line is for CSM

value, their p-mode frequencies are in poor agreement with the observations, and their initial helium abundance adjusted by matching the model's radius and luminosity to the observed values at the present solar age is too small. However, much evidence indicates that the Sun may be enriched with heavy elements, and the metal abundance in its convection zone can be higher than in the radiative core.
We investigate this possibility again, using up-to-date input physics and some improvements. We do not adopt a very low central metal abundance, which has often been used to considerably reduce the predicted solar neutrino flux. Instead, we calculate a model with the central metal abundance just a little lower than the surface value. It is interesting to note that our metal enriched model results in adequate depth of the convection zone and appropriate surface helium abundance, both of which are in good agreement with the seismic results. The agreement between the p-mode frequencies of our metal enriched models and the observations are also improved, and even better than the standard homogeneous solar model, SSM. The sound speed of our model CSM is worse than SSM but that of DSM is better than SSM in the area where $R<0.6 R_{\odot}$. It seems that element diffusion can reduce the difference of the sound speed between calculated and observed in the solar interior. In the base of the convective envelope, our model gives the sound speed more accurately than that of DSM and SSM. Further more, our metal-enriched model CSM gives lower neutrino fluxes for either $\mathrm{Cl}$ or $\mathrm{Ga}$ detectors than do SSM and DSM, which is helpful to solve the solar neutrino problem.

Investigations based on the solar neutrino experiments made by Fukugita \& Hata (1998) set an important restriction on the metal abundance distribution over the solar interior, i.e. the ratio of the metal abundance in the solar core to that in the solar envelope is in the range of $0.4-1.4$, with the peak of the probability at 0.8 . This clearly shows 
that the central metal abundance can be smaller than the surface metal abundance of the Sun.

Given the improvements discussed above, we conclude that solar models with an enhancement of heavy elements exterior to the base of their convection zones are still useful, and we cannot rule out either DSM or CSM with present helioseismological analysis.

Acknowledgements. This work are co-sponsored by the NSFC of China through project number 19625306 and 19833040 and National Key Fundamental Research Project "Formation and Evolution of Galaxies". Fruitful discussions with R. Q. Huang, G. Q. Luo, and Z. W. Han are highly appreciated.

\section{References}

Alexander, D. R., \& Ferguson, J. W. 1994, ApJ, 437, 879

Anders, E., \& Grevesse, N. 1989, Geochim. Cosmochim. Acta, 53, 197

Bahcall, J. N. 1989, Neutrino Astrophysics (Cambridge Univ. Press, Cambridge)

Bahcall, J. N., \& Ulrich, R. K. 1971, ApJ, 170, 593

Bahcall, J. N., \& Ulrich, R. K. 1988, Rev. Mod. Phys., 60, 297

Bahcall, J. N., \& Pinsonneault, M. H. 1992, Rev. Mod. Phys., 64, 885

Bahcall, J. N., Pinsonneault, M. H., \& Wasserburg, G. J. 1995, Rev. Mod. Phys., 67, 781

Bahcall, J. N., Pinsonneault, M. H., Basu, S., et al. 1997, Phys. Rev. Lett., 78, 171

Bahcall, J. N., Basu, S., \& Pinsonneault, M. H. 1998, Phys. Lett., B 433, 1

Basu, S., \& Antia, H. M. 1995, MNRAS, 276, 1402

Basu, S., \& Antia, H. M. 1997, MNRAS, 287, 189
Basu, S., Pinsonneault, M. H., \& Bahcall, J. N. 2000, ApJ, 529,1084

Christensen-Dalsgaard, J., \& Gough, D. O. 1980, Nature, 288, 544

Christensen-Dalsgaard, J., Gough, D. O., \& Morgan, J. G. 1979, A\&A, 73, 121

Cox, J. P., \& Giuli, R. T. 1968, Principles of Stellar Structure (Gordon Breach, New York)

Dar, A., \& Shaviv, G. 1996, ApJ, 468, 933

Fukugita, M., \& Hata, N. 1998, ApJ, 499, 513

Grevesse, N., \& Noels, A. 1993, Origin and evolution of the elements, ed. N. Prantzos, E. Vangioni, \& M. Cassé (Cambridge Univ. Press, Cambridge), 15

Henney, C. J., \& Ulrich, R. K. 1998, Effects of cometary mass accretion with heavy-element diffusion on solar oscillation frequencies, Structure and dynamics of the interior of the Sun and Sun-like stars, Proceedings of SOHO 6/GONE 98 Workshop, 473

Iglesias, C. A., \& Rogers, F. J. 1996, ApJ, 464, 943

Jeffery, C. S., Bailey, M. E., \& Chambers, J. E. 1997, Observatory, 117, 224

Joss, P. C. 1974, ApJ, 191, 771

Levy, E. H., \& Ruzmaikina, T. V. 1994, ApJ, 431, 881

Li, Y. 1992, A\&A, 257, 133

Libbrecht, K. G., Woodard, M. F., \& Kaufman, J. M. 1990, ApJS, 74, 1129

Rogers, F. J. 1994, The Equation of State in Astrophysics, ed. G. Chabrier, \& E. Schatzman, IAU Colloq., 147, 16

Rogers, F. J., \& Iglesias, C. A. 1995, ASP Conf. Ser., 78, 78

Rogers, F. J., Swenson, F. J., \& Iglesias, C. A. 1996, ApJ, 456, 902

Safronov, V. S., \& Ruzmaikina, T. V. 1985, in Protostars and Planets, ed. D. C. Black, \& M. S. Mathews, 959

Thoul, A. A., Bahcall, J. N., \& Loeb, A. 1994, ApJ, 421, 828 